\title{
Serum Glypican-3, Vascular Endothelial Growth Factor, and Interleukin-6 Levels in Hepatocellular Carcinoma
}

\author{
Murat SERILMEZ, ${ }^{1}$ Filiz AKYÜZ, ${ }^{2}$ Aslı ÖRMECi ÇiFTÇiBAŞI, ${ }^{2}$ Senem KARABULUT, ${ }^{3}$ \\ Sabahattin KAYMAKOĞLU, ${ }^{2}$ Vildan YASASEVER, ${ }^{1}$ Derya DURANYILDIZ' \\ 'Department of Basic Oncology, Istanbul University Institute of Oncology, Cancer Biochemistry, Istanbul-Turkey \\ ${ }^{2}$ Department of Gastroenterology, Istanbul Faculty of Medicine, Internal Medicine, Istanbul-Turkey \\ ${ }^{3}$ Department of Clinical Oncology, Istanbul University Institute of Oncology, Istanbul-Turkey
}

\section{OBJECTIVE}

The current study was an investigation of the serum levels of vascular endothelial growth factor (VEGF), interleukin 6 (IL-6), and glypican 3 (GPC-3), as potential diagnostic and prognostic indicators in cirrhotic patients and patients with hepatocellular carcinoma (HCC) or who are predisposed to develop HCC.

\section{METHODS}

A total of 54 HCC patients and 30 patients with cirrhosis were enrolled in this study. The pretreatment serum level of GPC-3, VEGF, and IL-6 was determined using an enzyme-linked immunosorbent assay. A control group of 21 healthy age- and sex-matched individuals was also included.

\section{RESULTS}

The serum VEGF level was statistically significantly higher in the HCC patients compared with the control group ( $\mathrm{p}=0.001)$. The serum IL-6 level was also higher in the HCC patients $(\mathrm{p}=0.002)$ and the cirrhotic patients $(\mathrm{p}=0.006)$ compared with the control group, with a statistically significant difference. There was also a statistically significant difference between patients with HCC and the patients with cirrhosis $(\mathrm{p}=0.001)$. Furthermore, the serum GPC-3 level was significantly higher in the HCC patients $(\mathrm{p}=0.009)$ and the cirrhotic patients ( $\mathrm{p}=0.001$ ) compared with the healthy control group.

\section{CONCLUSION}

To our knowledge, this is the first study to determine the serum values of these parameters in patients with HCC and cirrhosis. They may be useful markers to help clinicians reach a diagnosis of HCC.

Keywords: GPC3; hepatocellular carcinoma; IL-6; VEGF.

Copyright@ 2018, Turkish Society for Radiation Oncology

\section{Introduction}

Hepatocellular carcinoma (HCC) is the primary type of liver cancer; both age-adjusted incidence and mortality of HCC have steadily increased in recent years. The prognosis of advanced HCC is very poor. When diagnosed, most cases have already exceeded the lim- its of tumor treatment. The major risk factors for HCC are viral (chronic hepatitis $\mathrm{B}$ and $\mathrm{C}$ ), toxic (alcohol and aflatoxins), metabolic (diabetes and nonalcoholic fatty liver disease, hereditary haemochromatosis), and immune-related diseases (primary biliary cirrhosis and autoimmune hepatitis).[1]

International consensus on a diagnostic pathway 
exists because there is no ideal screening modality. Serum alpha fetoprotein (AFP) level is the most commonly used serological test in conjunction with hepatic ultrasonography to detect HCC in cirrhotic patients. A number of serum markers [e.g., AFP, hepatocyte growth factor (HGF), transforming growth factor beta 1 (TGFB-1), and serum proteomics] have been proposed, and several of these markers have been promising for detecting HCC in the clinical setting. Future research may show more specific and sensitive markers using proteomic or metabolomic approaches to screen blood or other biological fluids, such as urine.[2]

Glypicans (GPCs) are released from the cell surface by a lipase to regulate the signaling of wnts, hedgehogs, fibroblast growth factors, and bone morphogenetic proteins. [3-5] In mammals, the GPC family comprises six members. [6] It has been detected in the placenta and fetal liver, but not in other adult organs. GPC3 belongs to a group of heparan sulfate proteoglycans bound to the outer surface of the cell membrane through a glycosylphosphatidylinositol anchor.[7] GPC3 has a role in regulating cell proliferation and survival during embryonic development by modulating the activity of various growth factors. It also acts as a tumor suppressor gene. GPC3 can differentiate between malignant and benign hepatic lesions. Therefore, it seems to be a potential biomarker for the early diagnosis of HCC. $[8,9]$

Angiogenesis, defined as the formation of new blood vessels from the existing vasculature, is an important process regulating the growth and development of malignancies, including HCC.[10] The extensive hypervascularity associated with HCC is thought to be partly driven by the proangiogenic factor vascular endothelial growth factor (VEGF). VEGF is an endothelial cell mitogen that initiates and promotes neovascularization and endothelial cell proliferation, and it was initially identified as a vascular permeability factor. VEGF has a major effect in regulating angiogenesis, and its expression has been shown to correlate with carcinogenesis. Furthermore, the invasiveness of certain HCC lesions has recently been associated with high levels of VEGF, thereby leading several authors to conclude that an important association exists between VEGF and prognosis for HCC. [11,12]

Interleukin-6 (IL-6) is one of the major inflammatory cytokines, and in several types of target cells, it affects a variety of biological responses, including changes in cell differentiation, growth, and apoptosis and induction of acute-phase responses.[13] IL-6 expression is induced in various cell types, including endothelial cells, hepatocytes, and Kupffer cells. An increasing body of evidence indicates a key role of the pleiotropic cytokine IL-6 in liver damage process (chronic hepatitis B, cirrhosis) and carcinogenesis [14]. IL-6 is also implicated in tumor growth, progression, metastasis, and immune evasion, suggesting the future possibility of treating cancers via the modulation of the IL-6 pathway.[14,15]

Early diagnosis of HCC with a simple blood test detecting the increased levels of GPC3, VEGF, and IL-6 would likely be effective for the prognosis and treatment of the disease. In the present study, we investigated whether these serum markers can be useful for clinicians in deciding the diagnosis of HCC. We aimed to investigate the variance of these parameters in cirrhotic patients as it is known that $90 \%$ of patients with HCC have liver cirrhosis on the ground, and liver failure affects the prognosis.

\section{Materials and Methods}

This study comprised 54 patients with HCC and 30 with liver cirrhosis who were referred from Istanbul Medical Faculty Department of Gastroenterology and 21 healthy controls. The median age at diagnosis was 60 years (range, 36-77), where males constituted the majority of the group (89\%). All the patients had history of cirrhosis without vascular invasion, portal vein thrombosis, and extrahepatic disease. According to AASLD guideline 2011, liver biopsy for HCC diagnosis is not necessary in cirrhotic patients whose liver nodules $(>1 \mathrm{~cm})$ fulfill the contrast-enhanced imaging criteria (hypervascular in the arterial phase with washout in the portal venosus or delayed phase) or whose AFP levels are $>200 \mathrm{ng} / \mathrm{mL}$. Liver biopsy was performed for only nine patients. Patients were staged according to the Child-Pugh score, Barcelona Clinic Liver Cancer (BCLC) staging system, and model for end-stage liver disease (MELD). Patients with HCC were divided into two groups: early stage (Child-Pugh and BCLC, A; MELD, <11) and advanced stage (Child-Pugh and BCLC, B or C; MELD, >11). Patients with HCC underwent various imaging modalities, such as computed tomography (CT), magnetic resonance imaging (MRI), and magnetic resonance cholangiopancreatography (MRCP). Blood samples of the patients were collected before any treatment was administered. The pretreatment evaluation included detailed clinical history and physical examination with biochemistry tests and complete blood cell counts. Outpatients with ECOG performance status $\leq 2$ and appropriate blood biochemis- 
try tests received ablative treatment (hepatic resection and/or radiofrequency ablation) or palliative treatment, such as $90 \mathrm{Y}$ radioembolization and/or transarterial chemoembolization. None of the patients received sorafenib. The response to treatment was evaluated according to internal criteria. Treatment was continued until disease progression or unacceptable toxicity.

For comparison of serum GPC3, VEGF, and IL-6 levels, 21 age- and sex-matched healthy controls were included in the analysis. Our study was approved by the Istanbul University Ethics Committee (2556-24). The protocol was consistent with the Declaration of Helsinki (1989). Informed consents were obtained from all the study participants.

Blood samples of the patient and control groups were obtained by venipuncture and clotted at room temperature. The sera were collected following centrifugation and immediately frozen at $-20^{\circ} \mathrm{C}$ until analysis.

\section{Measurement of serum GPC3, VEGF, and IL-6 levels} GPC3 enzyme-linked immunosorbent assay (ELISA; USCN Life Science Inc., Wuhan P.R., China) is a sandwich enzyme immunoassay employing monoclonal antibodies. An antibody specific for human GPC3 was immobilized onto the surface. The sample and biotinylated detector monoclonal antibody were pipetted into the wells and allowed to incubate for $2 \mathrm{~h}$. GPC3 binds to the capture and detection of antibodies. Unbound GPC3 was washed away. Next, horseradish peroxidaseconjugated streptavidin was added, which bound to the detector antibody. Horseradish peroxidase catalyzes the conversion of the chromogenic substrate tetramethylbenzidine (TMB) from a colorless to blue solution (or yellow after the addition of stopping reagent), the intensity of which is proportional to the amount of GPC3 in the sample. The colored reaction product was measured using an automated ELISA reader (Rayto, RT-1904C Chemistry Analyzer, Atlanta GA, USA). The results were expressed as $\mathrm{ng} / \mathrm{mL}$.

VEGF assay (Invitrogen Corporation, Camarillo, USA) employs the quantitative sandwich enzyme immunoassay technique. A monoclonal antibody specific for VEGF was precoated onto a microplate. Standards and samples were pipetted into the wells, and any VEGF present was bound by the immobilized antibody. After washing any unbound VEGF, an enzymelinked polyclonal antibody for VEGF was added to the wells. Following a wash for removing any unbound antibody-enzyme-reagent, a substrate solution was added to the wells. Color development is proportion-

\begin{tabular}{|c|c|}
\hline Variables & $\mathbf{n}$ \\
\hline No. of patients & 54 \\
\hline \multicolumn{2}{|l|}{ Age of patients } \\
\hline \multicolumn{2}{|c|}{ Median (min-max): 60 (36-77) } \\
\hline$-60 / 61+$ & $30 / 24$ \\
\hline \multicolumn{2}{|l|}{ Sex } \\
\hline Male/Female & $48 / 6$ \\
\hline \multicolumn{2}{|l|}{ Smoking } \\
\hline Yes/No & $34 / 20$ \\
\hline \multicolumn{2}{|l|}{ Alcohol usage } \\
\hline Yes/No & $19 / 35$ \\
\hline \multicolumn{2}{|l|}{ HBV infection } \\
\hline Yes/No & $38 / 16$ \\
\hline \multicolumn{2}{|l|}{ HCV infection } \\
\hline Yes/No & $12 / 42$ \\
\hline \multicolumn{2}{|l|}{ Anti-viral therapy } \\
\hline Yes/No & $29 / 25$ \\
\hline \multicolumn{2}{|l|}{ Cirrhosis etiology } \\
\hline Viral/Non-viral & $46 / 8$ \\
\hline
\end{tabular}

Table 2 Cirrhotic patients characteristics

\begin{tabular}{lcc} 
& $\mathbf{n}$ & $\%$ \\
\hline No. of patients & 30 & 100 \\
Age of patients & $55.0 \pm 8.0$ & - \\
Sex Male/Female & $21 / 9$ & $70 / 30$ \\
HBV infection/HCV infection & $25 / 8$ & $83.3 / 26.7$ \\
HBV+HCV infection & 3 & 10 \\
Alcohol/Smoking & $17 / 19$ & $56.7 / 63.3$ \\
Alcohol+Smoking & 6 & 20 \\
\hline
\end{tabular}

al to the amount of VEGF bound in the initial step. Color development was stopped, and the intensity of the color was measured using an ELISA reader (Rayto, RT-1904C Chemistry Analyzer). The results were expressed as $\mathrm{ng} / \mathrm{mL}$.

IL-6 (Invitrogen Corporation) levels were determined using specific ELISA. IL- 6 anti- monoclonal coating antibody was adsorbed onto the microwells. IL-6 present in the sample or standard bound to antibodies adsorbed onto the microwells. Next, an FITCconjugated monoclonal anti-IL-6 antibody was added, and IL- 6 bound to the IL- 6 adsorbed onto the first antibody. Following incubation, anti-IL-6 was washed, and HRP-conjugated antibody was added and immobilized by the sandwich. Following incubation, unbound antiFITC-HRP was washed, and substrate solution reactive with HRP was added to the wells. A colored product was formed proportional to the amount of IL- 6 present in the sample. The reaction was terminated by the ad- 


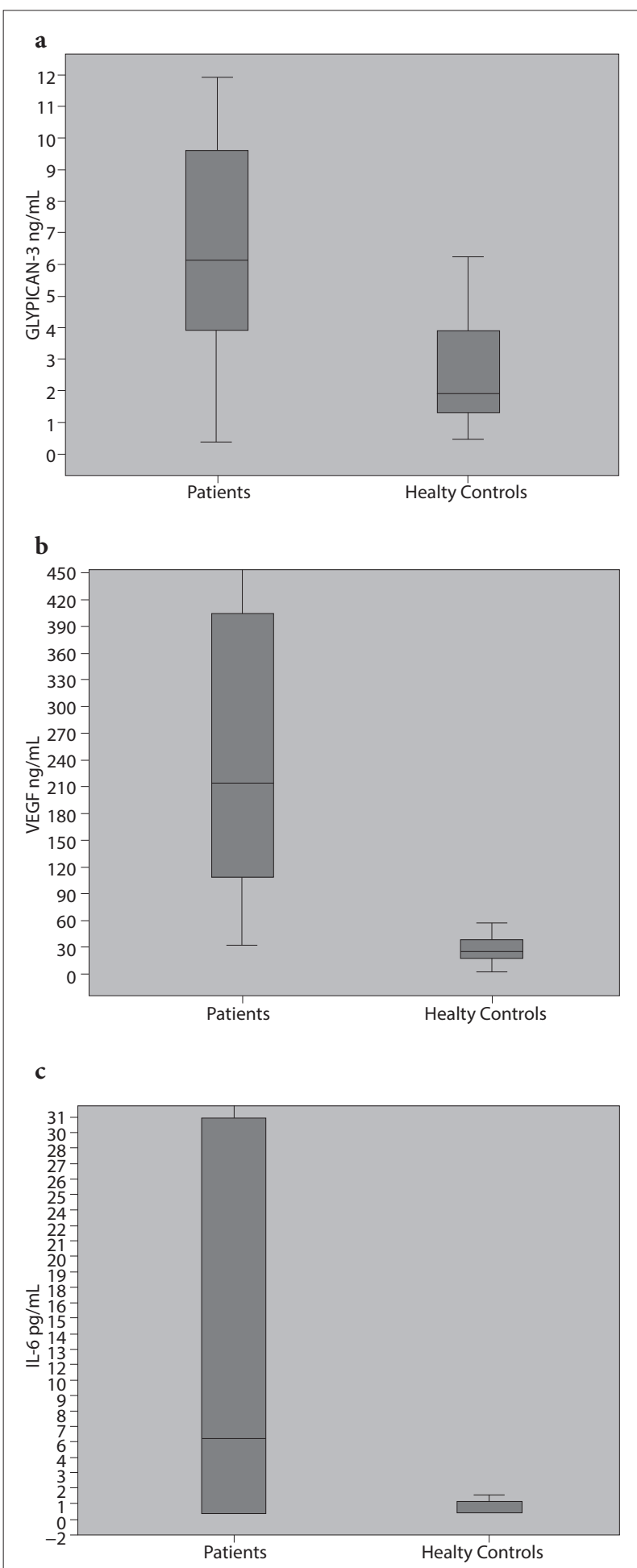

Fig. 1. (a) The values of serum GPC3 assays in patients with HCC and healthy controls $(\mathrm{p}<0.001)$. (b) The values of serum VEGF assays in patients with HCC and healthy controls ( $\mathrm{p}<0.001)$. (c) The values of serum IL- 6 assays in patients with HCC and healthy controls $(\mathrm{p}=0.02)$.
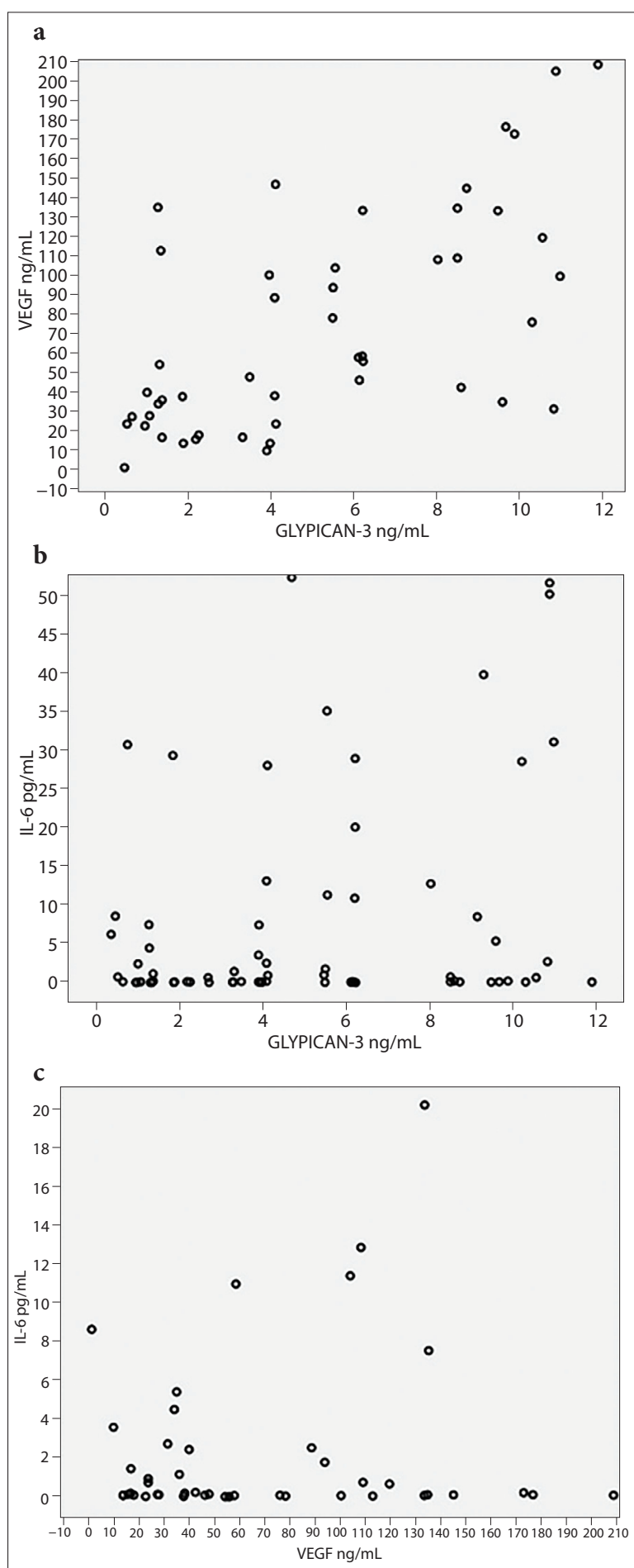

Fig. 2. (a) Correlation between serum GPC3 and VEGF levels in patients with HCC ( $r s=0.269, n=54$, $\mathrm{p}=0.02$; Spearman's correlation). (b) Correlation between serum GPC3 and IL-6 levels in patients with HCC ( $\mathrm{rs}=0.257, \mathrm{n}=54, \mathrm{p}=0.02$; Spearman's correlation). (c) Correlation between serum VEGF and IL-6 levels in patients with HCC ( $r s=0.416$, $\mathrm{n}=54, \mathrm{p}<0.001$; Spearman's correlation). 
dition of a stop solution, and absorbance was measured using an ELISA reader (Rayto, RT-1904C Chemistry Analyzer).The results were expressed as $\mathrm{pg} / \mathrm{mL}$.

\section{Statistical analysis}

SPSS software (version 16; SPSS, Chicago, IL) was used for statistical analysis. The data did not show a normal distribution; therefore, the nonparametric MannWhitney $U$ test was used to evaluate the differences between patients and normal controls. A two-tailed pvalue of $<0.05$ was considered statistically significant. The report design was adopted from the Standards for Reporting Diagnostic Accuracy (STARD) group (Bossuyt et al. 2004). Survival was calculated from the date of first hospital admission to death resulting from any cause or to last contact with the patient or any family member. The Kaplan-Meier method was used for the estimation of survival distribution and log-rank statistics for differences in survival. A p-value of $<0.05$ was considered statistically significant. The sensitivity and specificity of the tests were calculated using receiver operating characteristics (ROC) curves.

\section{Results}

Histopathological characteristics and demographic features of patients are listed in Tables 1 and 2, respectively. A total of $46 \%(n=25)$ of patients had ChildPugh score A, 30\% $(n=16)$ had B or $C$, and scores for $24 \%(n=13)$ of patients were unknown. The levels of serum GPC3, VEGF, IL-6, and AFP in patients with HCC, those with cirrhosis, and healthy controls are shown in Table 3. The baseline serum GPC3 levels were significantly higher in patients with HCC (6.1 vs. $1.9 \mathrm{ng} / \mathrm{mL} ; \mathrm{p}<0.001)$ and those with cirrhosis $(5.9$ vs $1.9 \mathrm{ng} / \mathrm{mL} ; \mathrm{p}=0.001$ ) than in controls (Fig. 1a). The se-

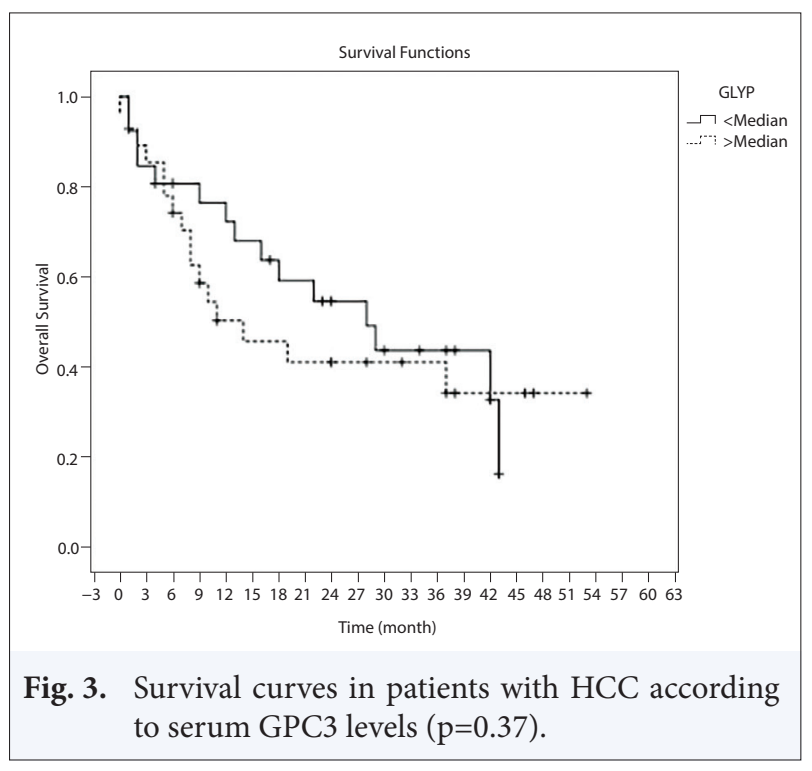

rum VEGF levels were significantly higher in patients with HCC (213.6 vs $24.4 \mathrm{ng} / \mathrm{mL} ; \mathrm{p}<0.001)$ and those with cirrhosis ( 118.3 vs $24.4 \mathrm{ng} / \mathrm{mL} ; \mathrm{p}=0.003$ ) than in controls (Fig. 1b). The serum IL-6 levels were significantly higher in patients with HCC (5.8 vs $0.2 \mathrm{pg} / \mathrm{mL}$; $\mathrm{p}=0.02)$ and those with cirrhosis $(2.3 \mathrm{vs} 0.2 \mathrm{pg} / \mathrm{mL}$; $\mathrm{p}=0.04$ ) than in the controls (Fig. 1c). The serum AFP levels were significantly higher in patients with HCC $(\mathrm{p}=0.001)$ and those with cirrhosis $(\mathrm{p}=0.002)$ than in controls. The correlation between GPC3 and VEGF levels was statistically significant ( $r s=0.269, n=54$, $\mathrm{p}=0.02$; Fig. 2a). The correlation between GPC3 and IL-6 levels was statistically significant $(r s=0.257, n=54$, $\mathrm{p}=0.02$; Fig. $2 \mathrm{~b}$ ). The correlation between VEGF and IL-6 levels was not statistically significant ( $r s=0.416$, $\mathrm{n}=54, \mathrm{p}>0.05$; Spearman's correlation; Fig. 2c).

The median follow-up duration was 14 months (range, 0-53 months). At the end of the observation period,

Table 3 The values of serum marker levels in patients with HCC, those with cirrhosis, and healthy controls

\begin{tabular}{|c|c|c|c|}
\hline & $\begin{array}{c}\text { HCC } \\
(n=54) \\
x \pm s d \\
\text { m(min,max) }\end{array}$ & $\begin{array}{l}\text { CIRRHOSIS } \\
(n=30) \\
x \pm s d \\
\text { m(min,max) }\end{array}$ & $\begin{array}{c}\text { CONTROL } \\
(n=21) \\
x \pm s d \\
\text { m(min,max) }\end{array}$ \\
\hline \multirow[t]{2}{*}{$\operatorname{VEGF}(\mathrm{pg} / \mathrm{mL})$} & $398.89 \pm 903.74$ & $160.3 \pm 155.8$ & $28.38 \pm 14.93$ \\
\hline & 213.55(32.10.6689.94) & $118.3(0.1-599.1)$ & $24.40(2.10 .56 .40)$ \\
\hline \multirow[t]{2}{*}{ IL-6 (pg/mL) } & $39.01 \pm 91.77$ & $4.0 \pm 4.3$ & $1.09 \pm 2.09$ \\
\hline & $5.84(0.06-437.89)$ & $2.3(0.1-13.7)$ & $0.17(0.05-8.61)$ \\
\hline \multirow[t]{2}{*}{ GPC-3 (ng/mL) } & $6.30 \pm 3.40$ & $6.0 \pm 3.0$ & $2.52 \pm 1.72$ \\
\hline & $6.11(0.40 .11 .85)$ & $5.9(0.2-9.5)$ & $1.91(0.50 .6 .22)$ \\
\hline \multirow[t]{2}{*}{ AFP $(\mathrm{pg} / \mathrm{mL})$} & $449.7 \pm 1043.5$ & $24.2 \pm 77.6$ & $2.8 \pm 1.4$ \\
\hline & 191.9(1.4-7010.0) & $8.7(2.8-434)$ & $2.5(0.7-6.7)$ \\
\hline
\end{tabular}




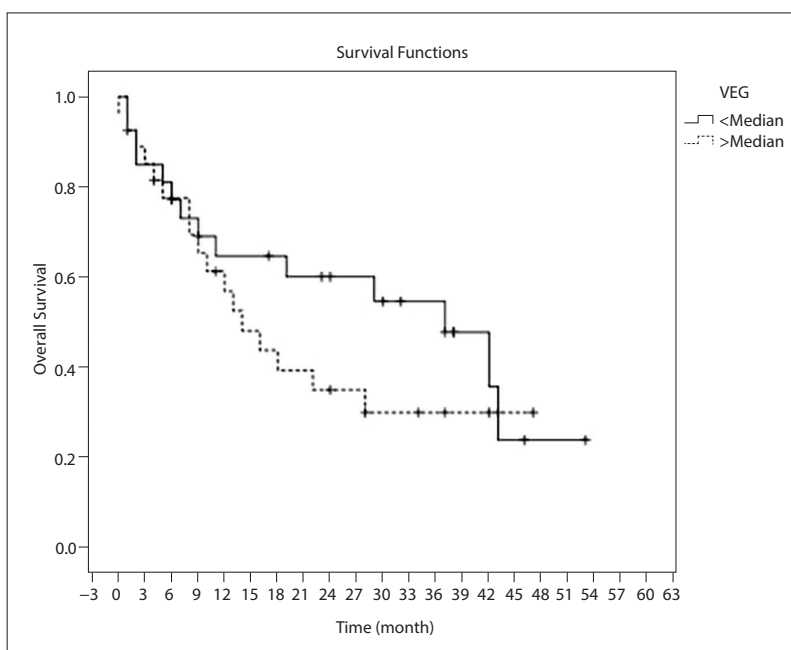

Fig. 4. Survival curves in patients with HCC according to serum VEGF levels $(\mathrm{p}=0.30)$.

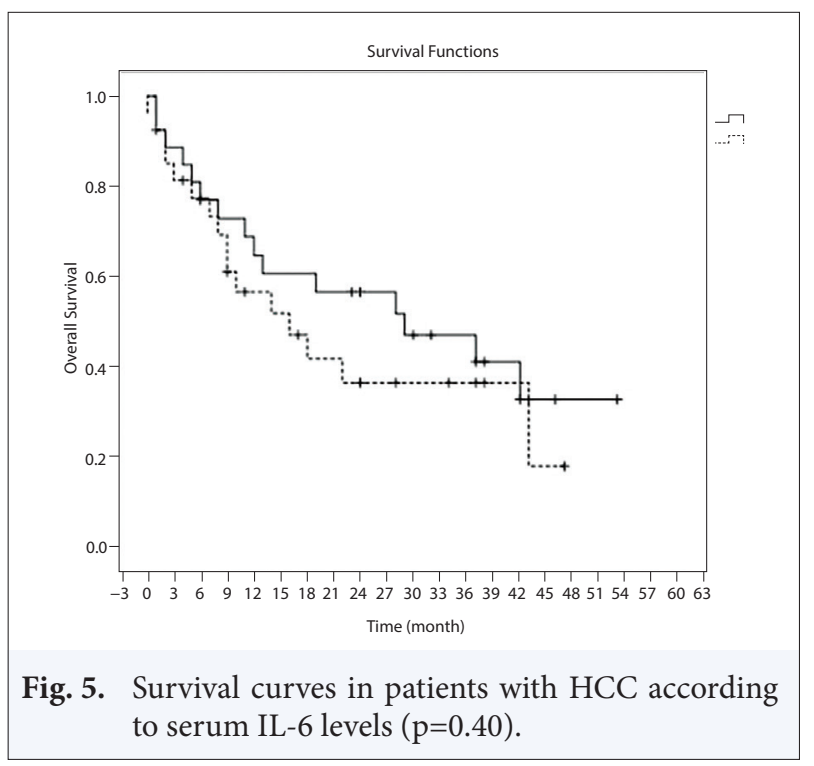

31 patients (57.4\%) were dead. The median survival of all the patients was $19.0 \pm 8.6$ months $(95 \% \mathrm{CI}=1.9-36.0$ months), where the 1-year overall survival rate was 63\% (95\% CI=49.7-76.3). Poor performance status $(\mathrm{p}<0.001)$, viral etiology of cirrhosis $(\mathrm{p}=0.03)$, larger tumor size $(\mathrm{p}=0.01)$, lower serum hemoglobin levels $(\mathrm{p}=0.03)$, and not treated for HCC $(\mathrm{p}=0.001)$ were related to worse survivals (Tables $4 \mathrm{a}$ and $4 \mathrm{~b}$ ). Serum GPC3, VEGF, and IL-6 levels were not found to have significant adverse effects on survival $(p=0.37, p=0.30$, and $\mathrm{p}=0.40$; Figures 3,4 , and 5 , respectively).

To determine the cut-off values and sensitivity and specificity of the tests, we used the ROC curves (Fig. 6). The cut-off values were chosen according to the ROC

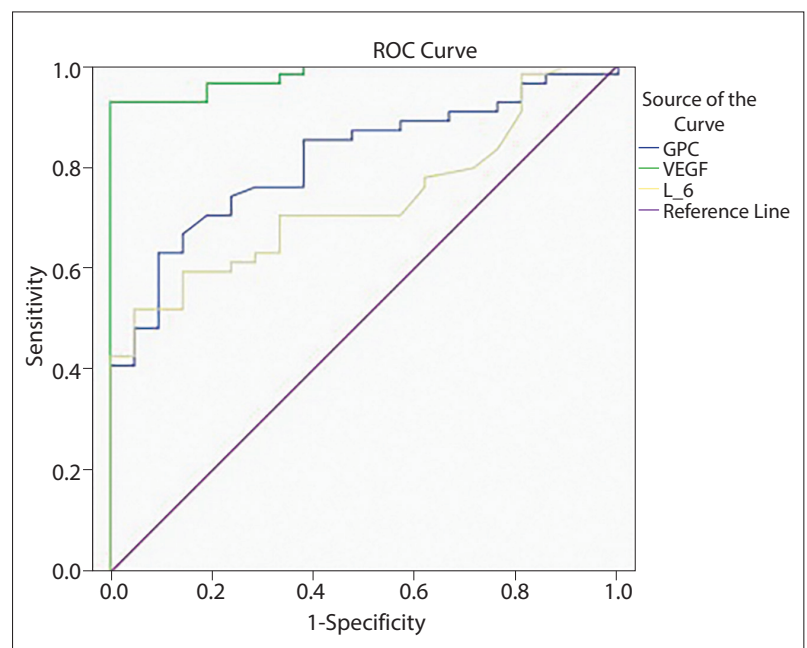

Fig. 6. ROC curve.

curve coordinate points, and cut-off points for serum VEGF, GPC3, and IL-6 were equal to their mean values. The cut-off levels $(\mathrm{x}+2 \mathrm{SD})$ for VEGF, GPC-3, and IL-6 were $58.24 \mathrm{pg} / \mathrm{mL}, 5.27 \mathrm{pg} / \mathrm{mL}$, and $5.96 \mathrm{ng} / \mathrm{mL}$, respectively.

The cut-off values were calculated using the ROC curve, which plots the ROC curve corresponding to the sensitivity and specificity of the test. The sensitivity and specificity values were as follows: VEGF, 100\% and $92.6 \%$; IL-6, 95.2\% and 46.3\%; GPC3, 90.5\% and $51.9 \%$, respectively.

\section{Discussion}

The major diagnostic parameters for HCC include serum markers, various imaging modalities, and histological analysis. A number of serum markers have been proposed, and several are currently used in common clinical practice for detecting HCC.[16] However, new markers for early diagnosis and better prediction of prognosis are required. In the present study, we evaluated the serum levels of GPC3, VEGF, and IL-6 in patients with HCC.

It was recently reported that GPC3 is present only in HCC cells but not in benign liver tissues and thus can be used as a potential biomarker for the early diagnosis of HCC. However, no correlation was observed between AFP and GPC3 levels, and only 53\% of patients with HCC had significantly elevated serum levels of GPC3. A subsequent study confirmed the presence of significantly increased serum GPC3 levels in patients with HCC. $[17,18]$ Consistent with these results, serum GPC3 levels of the HCC group were significant- 
Table 4a Survival analyses of clinical characteristics

\begin{tabular}{|c|c|c|c|}
\hline & $\begin{array}{c}\text { Median } \\
\text { survival time } \\
\text { (month) }( \pm S D)\end{array}$ & $\begin{array}{c}\text { 1-year } \\
\text { survival rate } \\
(\%)( \pm S D)\end{array}$ & $\mathbf{p}$ \\
\hline \multicolumn{4}{|c|}{ Age of patients } \\
\hline$<60$ & $18.0(9.1)$ & $69.7(8.5)$ & 0.66 \\
\hline$>60$ & $22.0(10.4)$ & $59.3(10.5)$ & \\
\hline \multicolumn{4}{|l|}{ Sex } \\
\hline Male & $19.0(8.4)$ & $62.9(7.2)$ & 0.94 \\
\hline Female & $43.0(0.0)$ & $66.7(19.2)$ & \\
\hline \multicolumn{4}{|c|}{ Performance status } \\
\hline $0-1$ & $37.0(7.3)$ & $78.2(7.3)$ & 0.001 \\
\hline $2-4$ & $5.0(1.8)$ & $30.8(12.8)$ & \\
\hline \multicolumn{4}{|l|}{ Smoking } \\
\hline Yes & $16.0(6.0)$ & $59.0(8.8)$ & 0.46 \\
\hline No & $29.0(12.1)$ & $52.0(11.7)$ & \\
\hline \multicolumn{4}{|l|}{ Alcohol usage } \\
\hline Yes & $16.0(4.9)$ & $47.4(11.5)$ & 0.63 \\
\hline No & $29.0(12.6)$ & $63.0(8.6)$ & \\
\hline \multicolumn{4}{|l|}{ HBV infection } \\
\hline Yes & $18.0(5.3)$ & $58.3(8.3)$ & 0.42 \\
\hline No & $28.0(10.2)$ & $73.4(11.5)$ & \\
\hline \multicolumn{4}{|l|}{ HCV infection } \\
\hline Yes & $13.0(3.7)$ & $64.3(14.6)$ & 0.58 \\
\hline No & $22.0(10.4)$ & $78.1(6.5)$ & \\
\hline \multicolumn{4}{|c|}{ Cirrhosis etiology } \\
\hline Viral & $23.1(3.2)$ & $54.0(7.8)$ & 0.03 \\
\hline Non-viral & $35.2(2.2)$ & No event & \\
\hline \multicolumn{4}{|c|}{ Viral ethiology of cirrhosis } \\
\hline HBV & $14.0(5.4)$ & $52.7(8.4)$ & 0.79 \\
\hline $\mathrm{HCV}$ & $13.0(5.3)$ & $62.5(21.3)$ & \\
\hline \multicolumn{4}{|l|}{ Tumor size } \\
\hline$<$ median & $31.3(4.1)$ & $67.9(10.0)$ & 0.01 \\
\hline$>$ median & $18.6(3.4)$ & $55.4(10.5)$ & \\
\hline \multicolumn{4}{|l|}{ Tumor style } \\
\hline Single & $22.0(10.1)$ & $53.1(10.9)$ & 0.67 \\
\hline Multiple & $29.0(13.4)$ & $63.4(10.3)$ & \\
\hline \multicolumn{4}{|l|}{ MELD score } \\
\hline$<11$ & $43.0(19.9)$ & $65.0(9.4)$ & 0.09 \\
\hline$>11$ & $13.0(8.8)$ & $53.4(14.1)$ & \\
\hline \multicolumn{4}{|c|}{ CHILD classification } \\
\hline A & $37.0(19.9)$ & $66.7(9.6)$ & 0.53 \\
\hline$B+C$ & $13.0(15.7)$ & $47.6(13.1)$ & \\
\hline \multicolumn{4}{|c|}{ Barcelona staging system } \\
\hline A & $28.0(12.9)$ & $59.7(9.9)$ & 0.39 \\
\hline$B+C$ & $13.0(4.1)$ & $60.6(10.9)$ & \\
\hline \multicolumn{4}{|l|}{ Treatment } \\
\hline Yes & $30.8(3.5)$ & $37.5(12.1)$ & 0.001 \\
\hline No & $14.2(4.2)$ & $3.5(7.6)$ & \\
\hline
\end{tabular}

Significant $\mathrm{p}$-values $(<0.05)$ are highlighted in bold.

ly higher than those of the control group in this study. Most studies have revealed that GPC3 is an impor-
Table 4b Survival analyses of laboratory parameters

\begin{tabular}{lccc} 
& $\begin{array}{c}\text { Median } \\
\text { survival time } \\
\text { (month) }(\mathbf{\pm S D})\end{array}$ & $\begin{array}{c}\text { 1-year } \\
\text { survival rate } \\
(\%)( \pm \text { SD) }\end{array}$ & p \\
\hline $\begin{array}{ccc}\text { Albumin (median) } \\
\text { Low }\end{array}$ & $25.7(4.1)$ & $74.1(8.4)$ & 0.86 \\
$\quad$ Normal & $26.1(0.0)$ & $61.5(9.5)$ & \\
AFP (median) & & & \\
$\quad$ Normal & $21.5(3.5)$ & $58.9(10.0)$ & 0.19 \\
$\quad$ Elevated & $31.0(4.8)$ & $65.3(10.0)$ & \\
GPC3 level(median) & & & \\
$\quad$ Normal & $28(7.5)$ & $76.5(8.4)$ & 0.37 \\
$\quad$ Elevated & $14.0(5.7)$ & $50.3(9.9)$ & \\
VEGF level (median) & & & \\
$\quad$ Normal & $37.0(10.5)$ & $64.7(9.5)$ & 0.30 \\
$\quad$ Elevated & $14.0(3.1)$ & $61.3(9.7)$ & \\
IL-6 level(median) & & & \\
$\quad$ Normal & $29.0(12.0)$ & $69.0(9.1)$ & 0.40 \\
Elevated & $16.0(5.6)$ & $56.9(9.9)$ & \\
\hline
\end{tabular}

Significant $\mathrm{p}$-values $(<0.05)$ are highlighted in bold.

tant tumor marker in the diagnosis of HCC. Taken together, our data demonstrated that GPC3 may be a more effective diagnostic marker than AFP for HCC because more frequent upregulation of GPC3 than of AFP was detected in patients with HCC.[17] To extend these findings, a recent large-scale study showed the potential of GPC3 in distinguishing liver adenoma and other benign hepatocellular conditions from well-differentiated HCC. Also, researchers have revealed that GPC3 could be used as an early marker for hepatic carcinogenesis as significantly increased levels of GPC3 were found in high-grade dysplastic or early HCC than in benign or low-grade dysplastic macronodules. Moreover, serum GPC3 levels measured using ELISA could be a useful tool in monitoring patients with HCC during follow-ups.[18] As a result, it can be concluded that GPC3 is an important marker for liver cancer, and recent data have proved its role in the diagnosis of hepatocellular neoplasms.[19,20]

High VEGF levels have been observed in patients with HCC with lesions of advanced pathological stage, vascular invasion, lack of capsule formation, thereby suggesting a strong relationship between VEGF and HCC prognosis.[21] In a recent study investigating the usefulness of serum VEGF level as an indirect marker for tumor levels using immunohistochemical staining of 60 specimens of resected HCC, it was found that VEGF level in hepatic tissue was in correlation with that in the platelets in circulation.[22] In 
the present study, the serum VEGF levels of patients with HCC were significantly higher than those in the control group, indicating a relationship between VEGF and HCC diagnosis. Our observations were supported by related recent studies which evaluated the impact of VEGF as a biomarker for HCC diagnosis. We found that VEGF had a sensitivity of $78 \%$ and specificity of $85 \%$ for HCC diagnosis, and significantly poor outcome was observed in patients with higher levels of serum VEGF. Interestingly, in a recent study, it was also reported that patients with higher levels of both angiogenic and anti-angiogenic factors had poorer survival. [23] The most noticeable factor associated with high levels of serum VEGF in patients with advanced HCC was hypoalbuminea, which is also known to be an independent risk factor in patients with HCC.

The level of serum IL-6 has been reported to be significantly elevated in patients with cholangiocarcinoma, hepatocellular carcinoma, and metastatic colorectal cancer compared with healthy controls and those with benign biliary diseases. [24] On the other hand, IL-6 may be responsible for liver inflammation and regeneration in chronic liver disease. IL-6 is also shown to induce the expression of mitogenic, motogenic, morphogenic, and pro-neoangiogenic scatter factors and HGF, which are commonly expressed at high levels in HCC.[25] Moreover, it is implicated in tumor growth, progression, metastasis, and invasion; therefore, the IL-6 pathway may be a possible target for cancer treatment in the future.[26,27]

Our results, which were in substantial agreement with some previous literature, indicated a potential role for IL-6 as a tumor marker for HCC. In particular, the diagnostic value of the test was significantly increased when it was used in association with AFP. Combining the two markers provided a new perspective in the diagnosis of HCC. Although the combined use of IL-6 and other serum biological markers was not found to have any prognostic value in a large cohort of patients with HCC, further trials are warranted to investigate its diagnostic and especially prognostic values to confirm its clinical usefulness for diagnosis and monitoring of patients with HCC.[28]

In conclusion, we evaluated the serum levels of GPC3, VEGF, and IL-6 in HCC patients of various clinical stages. We found that these markers had diagnostic values but could not identify any prognostic value. We determined higher serum levels of GPC3 in patients with HCC who had increased serum levels of AFP. In addition, GPC3 acts as an oncofetal protein, such as AFP; therefore, combined use of serum
GPC3 and AFP levels may be an important marker for the diagnosis of HCC. We believe that GPC3 can be an important marker when used with FAP in HCC diagnosis. Moreover, in patients who have another accompanying disease that causes elevated AFP levels, we suggest that the evaluation of GPC3 in association with AFP may help in the diagnosis of HCC in the clinical practice. The determination of serum GPC-3 levels can be combined with radiodiagnostic methods.

Further studies with larger sample sizes are needed to determine the potential clinical significance of these markers in HCC diagnosis. Furthermore, additional studies are required to investigate if their roles can be extended to design targeted therapies for cancer.

\section{Conclusion}

In conclusion, we evaluated the serum levels of GPC3, VEGF, and IL-6 in HCC patients of various clinical stages. We found that these markers had diagnostic values but could not identify any prognostic value. We determined higher serum levels of GPC3 in patients with HCC who had increased serum levels of AFP. In addition, GPC3 acts as an oncofetal protein, such as AFP; therefore, combined use of serum GPC3 and AFP levels may be an important marker for the diagnosis of HCC. We believe that GPC3 can be an important marker when used with FAP in HCC diagnosis. Moreover, in patients who have another accompanying disease that causes elevated AFP levels, we suggest that the evaluation of GPC3 in association with AFP may help in the diagnosis of HCC in the clinical practice. The determination of serum GPC-3 levels can be combined with radiodiagnostic methods. Further studies with larger sample sizes are needed to determine the potential clinical significance of these markers in HCC diagnosis. Furthermore, additional studies are required to investigate if their roles can be extended to design targeted therapies for cancer.

Acknowledgment: This study was supported by I. U. Research Fund (4973) (Local Ethical Committee, Number: 2556-24).

This article was reviewed by a biostatistician from I.U. Oncology Institute, Preventive Oncology, Biostatistics and Epidemiology Department.

Peer-review: Externally peer-reviewed.

Conflict of Interest: The authors declare that they have no conflicts of interest. 
Authorship contributions: Concept - M.S.; Design - M.S. S.K.; Supervision - D.D., V.Y.; Materials - A.Ö.Ç.; Data collection \&/or processing - F.A., S.K., A.Ö.Ç.; Analysis and/ or interpretation - S.K., D.D.; Literature search - M.S., D.D.; Writing - M.S.; Critical review - D.D., F.A.

List of abbreviations:

HCC: Hepatocellular carcinoma

GPC3: Gypican-3

VEGF: Vascular endothelial growth factor

IL-6: Interleukin-6

AFP: Alpha fetoprotein

HGF: Hepatocyte growth factor

TGFB-1: Transforming growth factor beta 1

BCLC: Barcelona Clinic Liver Cancer

MELD: Model For End-Stage Liver Disease

AASLD: American Association for the Study of Liver Diseases.

CT: Computed tomography

MRI: Magnetic resonance imaging

MRCP: Magnetic resonance cholangiopancreatography

\section{References}

1. Hamed MA, Ali SA. Non-viral factors contributing to hepatocellular carcinoma. World J Hepatol 2013;5(6):311-22. [CrossRef]

2. Gomaa AI, Khan SA, Leen EL, Waked I, Taylor-Robinson SD. Diagnosis of hepatocellular carcinoma. World J Gastroenterol 2009;15(11):1301-14. [CrossRef]

3. Song HH, Shi W, Xiang YY, Filmus J. The loss of glypican-3 induces alterations in Wnt signaling. J Biol Chem 2005;280(3):2116-25. [CrossRef]

4. Stigliano I, Puricelli L, Filmus J, Sogayar MC, Bal de Kier Joffé E, Peters MG. Glypican-3 regulates migration, adhesion and actin cytoskeleton organization in mammary tumor cells through Wnt signaling modulation. Breast Cancer Res Treat 2009;114(2):251-62.

5. Torisu Y, Watanabe A, Nonaka A, Midorikawa Y, Makuuchi M, Shimamura T, et al. Human homolog of NOTUM, overexpressed in hepatocellular carcinoma, is regulated transcriptionally by beta-catenin/TCF. Cancer Sci 2008;99(6):1139-46. [CrossRef]

6. Filmus J. The contribution of in vivo manipulation of gene expression to the understanding of the function of glypicans. Glycoconj J 2002;19(4-5):319-23. [CrossRef]

7. Shirakawa H, Suzuki H, Shimomura M, Kojima M, Gotohda N, Takahashi S, et al. Glypican-3 expression is correlated with poor prognosis in hepatocellular carcinoma. Cancer Sci 2009;100(8):1403-7. [CrossRef]

8. Capurro M, Wanless IR, Sherman M, Deboer G, Shi W, Miyoshi E, et al. Glypican-3: a novel serum and histochemical marker for hepatocellular carcinoma. Gastroenterology 2003;125(1):89-97. [CrossRef]

9. Nakatsura T, Yoshitake Y, Senju S, Monji M, Komori H, Motomura Y, et al. Glypican-3, overexpressed specifically in human hepatocellular carcinoma, is a novel tumor marker. Biochem Biophys Res Commun 2003;306(1):16-25. [CrossRef]

10. Zhou L, Liu J, Luo F. Serum tumor markers for detection of hepatocellular carcinoma. World J Gastroenterol 2006;12(8):1175-81. [CrossRef]

11. Kanda M, Nomoto S, Nishikawa Y, Sugimoto H, Kanazumi N, Takeda S, et al. Correlations of the expression of vascular endothelial growth factor B and its isoforms in hepatocellular carcinoma with clinico-pathological parameters. J Surg Oncol 2008;98(3):190-6.

12. Li XM, Tang ZY, Qin LX, Zhou J, Sun HC. Serum vascular endothelial growth factor is a predictor of invasion and metastasis in hepatocellular carcinoma. J Exp Clin Cancer Res 1999;18(4):511-7.

13. Scheller J, Ohnesorge N, Rose-John S. Interleukin-6 trans-signalling in chronic inflammation and cancer. Scand J Immunol 2006;63(5):321-9. [CrossRef]

14. Cheng KS, Tang HL, Chou FT, Chou JW, Hsu CH, Yu CJ, et al. Cytokine evaluation in liver cirrhosis and hepatocellular carcinoma. Hepatogastroenterology 2009;56(93):1105-10.

15. Hsia CY, Huo TI, Chiang SY, Lu MF, Sun CL, Wu JC, et al. Evaluation of interleukin-6, interleukin-10 and human hepatocyte growth factor as tumor markers for hepatocellular carcinoma. Eur J Surg Oncol 2007;33(2):208-12. [CrossRef]

16. Grizzi F, Franceschini B, Hamrick C, Frezza EE, Cobos E, Chiriva-Internati M. Usefulness of cancer-testis antigens as biomarkers for the diagnosis and treatment of hepatocellular carcinoma. J Transl Med 2007;5:3.

17. Capurro M, Wanless IR, Sherman M, Deboer G, Shi W, Miyoshi E, et al. Glypican-3: a novel serum and histochemical marker for hepatocellular carcinoma. Gastroenterology 2003;125(1):89-97. [CrossRef]

18. Hippo Y, Watanabe K, Watanabe A, Midorikawa Y, Yamamoto $S$, Ihara $S$, et al. Identification of soluble $\mathrm{NH} 2$-terminal fragment of glypican-3 as a serological marker for early-stage hepatocellular carcinoma. Cancer Res 2004;64(7):2418-23. [CrossRef]

19. Wang XY, Degos F, Dubois S, Tessiore S, Allegretta M, Guttmann RD, et al. Glypican-3 expression in hepatocellular tumors: diagnostic value for preneoplastic lesions and hepatocellular carcinomas. Hum Pathol 2006;37(11):1435-41 [CrossRef]

20. Yamauchi N, Watanabe A, Hishinuma M, Ohashi K, Midorikawa Y, Morishita Y, et al. The glypican 3 oncofetal protein is a promising diagnostic marker for hepatocellular carcinoma. Mod Pathol 2005;18(12):1591-8.

21. Amaoka N, Osada S, Kanematsu M, Imai H, Tomita H, 
Tokuyama Y, et al. Clinicopathological features of hepatocellular carcinoma evaluated by vascular endothelial growth factor expression. J Gastroenterol Hepatol 2007;22(12):2202-7. [CrossRef]

22. Poon RT, Lau CP, Cheung ST, Yu WC, Fan ST. Quantitative correlation of serum levels and tumor expression of vascular endothelial growth factor in patients with hepatocellular carcinoma. Cancer Res 2003;63(12):3121-6.

23. Sharma BK, Srinivasan R, Kapil S, Singla B, Saini N, Chawla YK, et al. Serum levels of angiogenic and anti-angiogenic factors: their prognostic relevance in locally advanced hepatocellular carcinoma. Mol Cell Biochem 2013;383(1-2):103-12. [CrossRef]

24. Goydos JS, Brumfield AM, Frezza E, Booth A, Lotze MT, Carty SE. Marked elevation of serum interleukin-6 in patients with cholangiocarcinoma: validation of util- ity as a clinical marker. Ann Surg 1998;227(3):398-404.

25. Zhu M, Paddock GV. Expression of the hepatocyte growth factor-like protein gene in human hepatocellular carcinoma and interleukin-6-induced increased expression in hepatoma cells. Biochim Biophys Acta 1999;1449(1):63-72. [CrossRef]

26. Malaguarnera M, Di Fazio I, Laurino A, Romeo MA, Giugno I, Trovato BA. Role of interleukin 6 in hepatocellular carcinoma. Bull Cancer 1996;83(5):379-84.

27. Giannitrapani L, Cervello M, Soresi M, Notarbartolo M, La Rosa M, Virruso L, et al. Circulating IL-6 and sIL-6R in patients with hepatocellular carcinoma. Ann N Y Acad Sci 2002;963:46-52. [CrossRef]

28. Parasole R, Izzo F, Perrone F, Pignata S, Galati MG, Leonardi E, et al. Prognostic value of serum biological markers in patients with hepatocellular carcinoma. Clin Cancer Res 2001;7(11):3504-9. 